\title{
Interband optical transitions in ellipsoidal shaped nanoparticles
}

\author{
Tamaz Kereselidze ${ }^{\mathrm{a}^{*}}$, Tamar Tchelidze $^{\mathrm{a}}$, Alexander Devdariani ${ }^{\mathrm{b}}$ \\ ${ }^{a}$ Faculty of Exact and Natural Sciences, Tbilisi State University, 0179 Tbilisi, \\ Georgia \\ ${ }^{\mathrm{b}}$ St. - Petersburg State University, St. Petersburg 198904, Russia
}

\begin{abstract}
The optical properties of crystalline semiconductor nanoparticles with ellipsoidal shape are investigated and discussed as a function of the shape-anisotropy parameter. The optical transitionmatrix elements are calculated in the dipole approximation using perturbation theory and with a direct diagonalization of the appropriate Hamiltonian. The matrix elements involving the ground and first excited states are monotonic functions of the shape-anisotropy parameter, whereas matrix elements involving the highly excited states have zeros and extrema that are reflected in the behaviour of the corresponding transition probabilities. Moreover, some matrix elements involving the excited states have discontinuity. We demonstrate that, nanoparticles with ellipsoidal shape can be grown with the infrared as well as ultraviolet features.
\end{abstract}

Keywords: nanoparticle, ellipsoidal shape, optical properties, transition-matrix elements

\section{Introduction}

The electronic and optical properties of crystalline semiconductor structures of various shapes and of nanometer size attract increasing interest [1]. In the regime of strong size quantisation, the optical properties are determined by the transitions between discrete states of electrons and holes confined in a nanoparticle, which signifies a particle of which the extent in each spatial dimension is of order 1 $\mathrm{nm}$. In most early publications, the qualitative and quantitative descriptions of the optical properties apply to spherically shaped nanoparticles, i.e. for spherical quantum dots (see [2] and references therein).

The first study [3] of the optical properties of a spherical quantum dot with infinitely high walls was a theoretical investigation of the direct absorption of light. The optical properties of nanoparticles having cylindrical, ellipsoidal, semiellipsoidal, pyramidal and lens shape were analysed in subsequent works [4-20]. The analysis of both the optical transition-matrix elements and the oscillator strengths in the dipole approximation revealed interesting features induced by the size and shape of a nanoparticle. Specifically, it was shown that the shape anisotropy plays a crucial role in determining the optical properties of nanoparticles. The most stimulating results were obtained in [7] in which a charged particle was considered to be in an ellipsoidally shaped potential well. The appropriate Schrödinger equation for an effective mass was separated in prolate spheroidal coordinates and the obtained one-dimensional equations were solved numerically for an ellipsoid arbitrarily deviating from a sphere, but transitions involving highly excited states have never been considered.

Experiments indicate that small nanoparticles have a nearly spherical shape, whereas large nanoparticles have an ellipsoidal shape [21]. For the growth of nanoparticles with various methods, the energy spectrum and, accordingly, the optical properties vary continuously with the size and shape of nanoparticle. It is important to recognise that nanoparticles of ellipsoidal shape have an advantage with respect to spherical quantum dots, which arises from the additional geometrical characteristics

\footnotetext{
*Corresponding author at: Faculty of Exact and Natural Sciences, Tbilisi State University, 0179 Tbilisi, Georgia.

E-mail address: tamaz.kereselidze@tsu.ge
} 
related to the shape-anisotropy parameter. That effect makes possible the tuning of the optical properties of objects of nanometre size. The tuneable control of spectral and optical characteristics of the nanometre objects through size and shape opens exciting possibilities for the engineering of new functional materials with a wide prospective application. Ellipsoidal nanoparticles thus play an important role for applications; the most promising candidates for further technological advances are precisely the ellipsoidally elongated objects of nanometre size. A comprehensive knowledge of the optical properties of ellipsoidally shaped nanoparticles is hence desirable.

In our recent paper [19], we considered a charged particle (electron or hole) confined in a potential well of ellipsoidal shape; the problem was solved in an effective-mass approximation. We assumed that a spherical potential well with infinitely high walls is subject to a small deformation, which makes its shape that of a slightly prolate ellipsoid. Because the deviation from the sphere is small, the problem is solvable according to perturbation theory, with the unperturbed wave functions and energy levels corresponding to a spherical well; the matrix elements for the operator describing a deviation from the sphere were obtained in an explicit algebraic form. The derived matrix elements enabled us to find the energy levels and the corresponding wave functions of a trapped particle in a high order of perturbation theory. In [19] the problem was solved also for an arbitrary deviation from a spherical shape using a direct diagonalization of the appropriate Hamiltonian. All calculations were undertaken algebraically; the obtained results are presented in an explicit form.

In the present work, we make use of the wave functions and energy levels obtained in [19] and calculate the dipole-moment matrix elements, the oscillator strengths and the optical transition probabilities to analyse the optical response of a nanoparticle when its shape alters from spherical to ellipsoidal. We consider a small deviation from a sphere; as well, we treat a case in which the deformation is not small. We pay special attention to transitions involving highly excited states. For the first time, we show that the dipole-moment matrix elements involving the ground and highly excited states have zeros, extrema and discontinuity.

The article is organized as follows. After stating the purpose, we present briefly the basic equations in Section 2. The results of calculations are presented and discussed in Section 3, before a conclusion in Section 4.

\section{Basic equations}

We consider a charged particle of effective mass $m^{*}$ confined in a potential well of ellipsoidal shape with semi-axes $a=b$ and $c ; a$ represents the size of a well in directions $x$ and $y, c$ is the size in direction $z$. The simplest potential $V(x, y, z)$ of that type is zero inside the ellipsoid and infinite on the surface of the ellipsoid and beyond. The corresponding Schrödinger equation that describes the motion of a particle trapped inside the ellipsoid, with the boundary condition for the wave function to be zero on the surface and outside the surface of the ellipsoid, reads

$$
\left[\Delta_{x y z}+K^{2}-\frac{2 m^{*}}{\hbar^{2}} V(x, y, z)\right] \Psi(x, y, z)=0,
$$

in which $K^{2}=2 m^{*} E / \hbar^{2}$.

We proceed to new variables $\xi=x r_{0} / a, \eta=y r_{0} / a$ and $\varsigma=z r_{0} / c$. In these variables the boundary condition transforms from the surface of the ellipsoid to the surface of the sphere of radius $r_{0}=\xi^{2}+\eta^{2}+\varsigma^{2}$. After writing the Laplace operator $\Delta_{x y z}$ in the new variables, we rewrite Eq. (1) as

$$
\left[\Delta_{\xi \eta \varsigma}+K^{2}-\alpha U(\xi, \eta, \varsigma)\right] \Psi(\xi, \eta, \varsigma)=0,
$$

in which

$$
U= \begin{cases}\frac{c^{2}\left(a^{2}-r_{0}^{2}\right)}{r_{0}^{2}\left(c^{2}-a^{2}\right)} \Delta_{\xi \eta \varsigma}+\frac{\partial^{2}}{\partial \varsigma^{2}} & \text { if } \xi^{2}+\eta^{2}+\varsigma^{2}<r_{0}, \\ \infty & \text { if } \xi^{2}+\eta^{2}+\varsigma^{2} \geq r_{0},\end{cases}
$$


and $\alpha=r_{0}^{2}\left(c^{2}-a^{2}\right) /(a c)^{2}$ is the parameter that reflects the deviation of the potential well from the spherical well.

We transform from coordinates $\xi, \eta$ and $\varsigma$ to spherical coordinates $\xi=r \sin \vartheta \cos \varphi$, $\eta=r \sin \vartheta \sin \varphi$ and $\varsigma=r \cos \vartheta$ in Eqs. (2) and (3), to obtain an equation that describes the motion of a particle in the sphere,

$$
\left[\Delta_{r \vartheta \varphi}+K^{2}-\alpha U(r, \vartheta, \varphi)\right] \Psi(r, \vartheta, \varphi)=0 .
$$

The problem of motion of a charged particle in an ellipsoidal well thus reduces to the solutions of Eq. (4), in which effective potential $U(r, \vartheta, \varphi)$ is defined with Eq. (3) with $\Delta_{\xi \eta \zeta}$ replaced with the Laplace operator written in spherical coordinates, and $\partial / \partial \varsigma=\partial / \partial r \cos \vartheta-r^{-1} \sin \vartheta \partial / \partial \vartheta$.

In the spherical potential well $(\alpha=0)$, the states of a particle are specified by the quantum numbers $n, l$ and $m$. Here $l$ is the orbital quantum number and $m=0, \pm 1, \pm 2, \ldots$ is the magnetic quantum number of the particle; $n$, which has no dependence on $m$, numbers the level in the spherical well for given $l$. The solutions of equation $\left(\Delta_{r \vartheta \varphi}+k_{n l}^{2}\right) \psi_{n l m}=0$ are well known and given in textbooks. The eigenfunctions are $\psi_{n l m}(r, \vartheta, \varphi)=B_{n l} j_{l}\left(k_{n l} r\right) Y_{l m}(\vartheta, \varphi) ; B_{n l}$ is a normalization factor, $j_{l}\left(k_{n l} r\right)$ are spherical Bessel functions and $Y_{l m}(\vartheta, \varphi)$ are spherical harmonics. The corresponding eigenvalues depend on the size of the potential well, $r_{0}$, and two quantum numbers $n$ and $l: k_{n l}^{2}=\tau_{n l}^{2} / r_{0}^{2}$, in which $\tau_{n l}$ is the $n$th root of the spherical Bessel function determined from the condition $j_{l}\left(k_{n l} r_{0}\right) \equiv j_{l}\left(\tau_{n l}\right)=0$.

\subsection{A perturbation approach}

We assume that the ellipsoid is almost a sphere of radius $r_{0}$. In this case, parameter $\alpha$ is small; Eq. (4) can accordingly be solved using perturbation theory. In [19], we found the first-, second- and third-order corrections to the unperturbed energy, $\varepsilon_{n l}=\hbar^{2} k_{n l}^{2} / 2 m^{*}$, and the first- and second-order corrections to the unperturbed wave function, $\psi_{n l m}$. The obtained results are presented in the appendix. Using Eq. (A.1) one can readily write the perturbed wave function in the second order of perturbation theory

$$
\begin{aligned}
& \Psi_{n l m}=\psi_{n l m}(r, \vartheta, \varphi)+\alpha\left[\chi_{n l+2}(r) Y_{l+2 m}(\vartheta, \varphi)\right. \\
& \left.+\chi_{n l-2}(r) Y_{l-2 m}(\vartheta, \varphi)\right]+\alpha^{2} \Psi_{n l m}^{(2)} .
\end{aligned}
$$

In (5), the perturbed wave functions $\Psi_{n l m}$ are specified with quantum numbers $n, l$ and $m$ that describe the states in the spherical potential well, $\chi_{l \pm 2}(r)$ denotes the sum

$$
\chi_{n l \pm 2}(r)=A_{l \pm 2} \sum_{n^{\prime}} \frac{\tau_{n l^{\prime}} \tau_{n^{\prime} l \pm 2}\left(\tau_{n l}^{2}-\tau_{n^{\prime} l \pm 2}^{2}\right)^{2}}{B_{n^{\prime} l \pm 2}} j_{l^{\prime \pm 2}}\left(k_{n^{\prime} l \pm 2} r\right),
$$

in which $A_{l \pm 2}$ are defined with Eq. (A.3).

We examine the optical properties of an ellipsoidal particle of which its shape differs slightly from spherical. For a spherical quantum dot, only transitions with $\Delta l= \pm 1$ and $\Delta m=0, \pm 1$ are allowed in the dipole approximation. The lesser degree of symmetry of an ellipsoidal nanoparticle relaxes the first selection rule, allowing transitions for which $\Delta l$ is odd, but the condition $\Delta m=0, \pm 1$ remains valid. The transitions with $\Delta m=0$ are associated with radiation linearly polarized along the $z$-axis; transitions with $\Delta m= \pm 1$ lead to radiation that is circularly polarized in the $x y$ plane. For the ellipsoidal nanoparticles, allowed transitions involving the ground state are thus $\langle 100|\leftrightarrow| n l m\rangle$ with $l=1,3,5, \ldots$ and $|m|=0,1$. The expression (5) obtained for the perturbed wave function shows that the dipole transition-matrix element is proportional to $\alpha^{0}$ for transition $|100\rangle \leftrightarrow|11 m\rangle$, to $\alpha$ for 
transition $|100\rangle \leftrightarrow|13 m\rangle$, to $\alpha^{2}$ for transition $|100\rangle \leftrightarrow|15 m\rangle$ and so forth. The transitions between the ground $|100\rangle$ and excited $|11 \mathrm{~m}\rangle$ states are hence the most intense for nanoparticles of ellipsoidal shape for which the deviation from the sphere is small.

After qualitative analysis, we proceed to derive the matrix elements of the electric-dipolar moment $\vec{d}=e \vec{r}$ with respect to the wave functions (5); $e$ is the charge and $\vec{r}$ is the position vector of a particle trapped in the ellipsoid. Specifically, we derive the matrix elements of operators $d_{ \pm}=2^{-1 / 2}(x \pm i y) / r_{0}$ and $d_{z}=z / r_{0}$, which determine the circularly and linearly polarized radiation, respectively. Because the integrals from the normalized spherical Bessel functions are proportional to $r_{0}$, the calculated matrix elements are independent of the size of the sphere for which the integration is implemented. The dependence of the matrix elements on shape-anisotropy parameter $c / a$ is expressible in the general form

$$
\left\langle\Psi_{n l m}\left|d_{ \pm, z}\right| \Psi_{n^{\prime \prime} l^{\prime} m^{\prime}}\right\rangle=g_{n l m, n^{\prime} l^{\prime} m^{\prime}}+\frac{r_{0}^{2}}{a^{2}}\left(1-\frac{a^{2}}{c^{2}}\right) q_{n l m, n^{\prime} l^{\prime} m^{\prime}}+O\left(\alpha^{2}\right),
$$

in which

$$
\begin{aligned}
& g_{n l m, n^{\prime} l^{\prime} m^{\prime}}=\left\langle\psi_{n l m}\left|d_{ \pm, z}\right| \psi_{n^{\prime} l^{\prime} m^{\prime}}\right\rangle, \\
& q_{n l m, n^{\prime} l^{\prime} m^{\prime}}=\left\langle\psi_{n l m}\left|d_{ \pm, z}\right| \chi_{n^{\prime} l^{\prime}+2} Y_{l^{\prime}+2 m^{\prime}}+\chi_{n^{\prime} l^{\prime}-2} Y_{l^{\prime}-2 m^{\prime}}\right\rangle \\
& +\left\langle\chi_{n l+2} Y_{l+2 m}+\chi_{n l-2 m} Y_{l-2 m}\left|d_{ \pm, z}\right| \psi_{n^{\prime} l^{\prime} m^{\prime}}\right\rangle,
\end{aligned}
$$

are the quantities that are independent on parameters $a, c$ and $r_{0}$. We note that $g_{n l m, n^{\prime} l^{\prime} m^{\prime}}=0$ for the transitions forbidden for the spherical quantum dot. Accordingly, $\left\langle\Psi_{n l m}\left|d_{ \pm, z}\right| \Psi_{n \prime \prime m}\right\rangle$ becomes zero when $c \rightarrow a$, i.e. when a nanoparticle has a spherical shape, and differs from zero when $q_{n l m, n^{\prime} l^{\prime} m^{\prime}} \neq 0$ and $c>a$. For some matrix elements involving the ground state, quantities $g_{100, n^{\prime}{ }^{\prime \prime} m^{\prime}}$ and $q_{100, n^{\prime} i^{\prime}}$ are collected in Table 1 . The upper quantities are $g_{100, n^{\prime} l^{\prime} m^{\prime}}$ and the lower quantities are $q_{100, n^{\prime} l^{\prime} m^{\prime}}$; the sign \pm corresponds to left and right circular polarization.

\section{Table 1}

Quantities $g_{100, \text { n' }^{\prime} \mathrm{m}^{\prime}}$ and $q_{100, \text {, }^{\prime \prime} \mathrm{m}^{\prime}}$ for some matrix elements involving the ground state

\begin{tabular}{|l|l|l|l|l|l|l|l|l|}
\hline & $n^{\prime}=1$ & $n^{\prime}=1$ & $n^{\prime}=2$ & $n^{\prime}=1$ & $n^{\prime}=1$ & $n^{\prime}=1$ & $n^{\prime}=2$ & $n^{\prime}=1$ \\
& $l^{\prime}=1$ & $l^{\prime}=3$ & $l^{\prime}=1$ & $l^{\prime}=5$ & $l^{\prime}=1$ & $l^{\prime}=3$ & $l^{\prime}=1$ & $i^{\prime}=5$ \\
& $m^{\prime}=0$ & $m^{\prime}=0$ & $m^{\prime}=0$ & $m^{\prime}=0$ & $m^{\prime}=\mp 1$ & $m^{\prime}=\mp 1$ & $m^{\prime}=\mp 1$ & $m^{\prime}=\mp 1$ \\
\hline $\begin{array}{l}l=1 \\
m=0\end{array}$ & 0.3060 & 0.0000 & -0.0226 & 0.0000 & $\mp 0.3060$ & 0.0000 & \pm 0.0226 & 0.0000 \\
& 0.0185 & 0.0093 & -0.0052 & 0.0000 & \pm 0.0093 & $\mp 0.0151$ & $\mp 0.0026$ & 0.0000 \\
\hline
\end{tabular}

\subsection{Diagonalization of the Hamiltonian}

When a deviation from the sphere is not small, the term $\alpha U$ cannot be considered a small perturbation; Eq. (4) can accordingly not be solved using perturbation theory. In this case we employ a diagonalization of the Hamiltonian, i.e. we reduce the solution of Eq. (4) to the solution of the appropriate system of linear homogeneous equations as in [19].

An analysis of the roots of the spherical Bessel functions yields the following series of the energy levels in the spherical well: $1 s, 1 p, 1 d, 2 s, 1 f, 2 p, 1 g, 2 d, 1 h, \ldots[22](s, p, d, \ldots$ denote 
the energy levels with the angular momentum quantum number $l=0,1,2, \ldots)$. For our purpose it is more convenient to consider the states with given $m$ separately and to enumerate the states in the following way: $\psi_{10}^{g} \equiv \psi_{100}(r, \vartheta, \varphi), \psi_{2 m}^{g} \equiv \psi_{12 m}(r, \vartheta, \varphi), \psi_{30}^{g} \equiv \psi_{200}(r, \vartheta, \varphi), \psi_{4 m}^{g} \equiv \psi_{14 m}(r, \vartheta, \varphi), \ldots$ and analogously $\quad \psi_{1 m}^{u} \equiv \psi_{11 m}(r, \vartheta, \varphi), \quad \psi_{2 m}^{u} \equiv \psi_{13 m}(r, \vartheta, \varphi), \quad \psi_{3 m}^{u} \equiv \psi_{21 m}(r, \vartheta, \varphi)$, $\psi_{4 m}^{u} \equiv \psi_{15 m}(r, \vartheta, \varphi), \ldots$ We hence introduce states in two sets with even and odd $l$. Taking into account that the matrix element $\left\langle\psi_{\text {nlm }}|U| \psi_{\text {nimm }^{\prime} m^{\prime}}\right\rangle$ differs from zero if $l^{\prime}=l \pm 2$ [19], one obtains that the introduced sets of wave functions are not bound; the states with even $(g)$ and odd $(u)$ parity can hence be considered independently.

We seek the solutions of Eq. (4) with a given parity and projection of the orbital momentum on the $z$-axis, $m$ as follows

$$
\Psi_{i m}^{g, u}(r, \vartheta, \varphi)=\sum_{j=1}^{4} C_{j m}^{g, u}\left(E_{i}\right) \psi_{j m}^{g, u}(r, \vartheta, \varphi) .
$$

Here $C_{j m}^{g, u}$ are expansion coefficients that depend on energy $E_{i}=\hbar^{2} K_{i}^{2} / 2 m^{*}$ of the $i$ th state; these coefficients are defined in the appendix. The consideration of only four states in (9) is stipulated by the fact that in this case the equation for the energy arises of the fourth degree, which can be solved algebraically using the Ferrari method [23]. The four-state approximation can reproduce with satisfactory accuracy all levels of an ellipsoidal nanoparticle that correspond to the spherical quantum dot levels specified at the beginning of this subsection (an exception is level $2 d$ with $m=0$, which should be treated in the five-state approximation). The behaviour of energy levels $E_{i} \equiv E_{n l m}=\hbar^{2} K_{n l m}^{2} / 2 m^{*}$ as a function of shape-anisotropy parameter $c / a$ is shown in Fig. 1. This figure demonstrates that the states with the same $n$ and $m$ but different $l$ become almost degenerate at the large values of $c / a$. When $a$ is fixed and $c$ increases infinitely these states thus create a miniband of the cylindrical quantum wire [16].

Taking into account that the dipole transitions occur between states of different parity, we obtain for the transition-matrix elements involving the ground state,

$$
\begin{aligned}
& \left\langle\Psi_{10}^{g}\left|d_{ \pm, z}\right| \Psi_{i m}^{u}\right\rangle=C_{10}^{g}\left(E_{1}\right)\left[\left\langle\psi_{100}\left|d_{ \pm, z}\right| C_{1 m}^{u}\left(E_{i}\right) \psi_{11 m}+C_{3 m}^{u}\left(E_{i}\right) \psi_{21 m}\right\rangle\right. \\
& +C_{20}^{g}\left(E_{1}\right)\left[\left\langle\psi_{120}\left|d_{ \pm, z}\right| C_{1 m}^{u}\left(E_{i}\right) \psi_{11 m}+C_{2 m}^{u}\left(E_{i}\right) \psi_{13 m}+C_{3 m}^{u}\left(E_{i}\right) \psi_{21 m}\right\rangle\right] \\
& +C_{30}^{g}\left(E_{1}\right)\left[\left\langle\psi_{200}\left|d_{ \pm, z}\right| C_{1 m}^{u}\left(E_{i}\right) \psi_{11 m}+C_{3 m}^{u}\left(E_{i}\right) \psi_{21 m}\right\rangle\right] \\
& +C_{40}^{g}\left(E_{1}\right)\left[\left\langle\psi_{140}\left|d_{ \pm, z}\right| C_{2 m}^{u}\left(E_{i}\right) \psi_{13 m}+C_{4 m}^{u}\left(E_{i}\right) \psi_{15 m}\right\rangle\right],
\end{aligned}
$$

in which $i=1,2,3,4$ and $m=\mp 1$. In Eq. (10), the integrals from the Bessel functions are calculated with tables of integrals [24].

We introduce the oscillator strength [25],

$$
F_{i f}=\frac{2 m^{*} r_{0}^{2}}{\hbar^{2}}\left(E_{f}-E_{i}\right)\left|\left\langle f\left|d_{ \pm, z}\right| i\right\rangle\right|^{2},
$$

which is dimensionless quantity that express the probability of emission and absorption of electromagnetic radiation in transitions between the energy levels $E_{i}$ and $E_{f}$ of a nanoparticle.

In the dipole approximation the transition probability during unit time from an initial state $|i\rangle$ to a final state $|f\rangle$ is proportional to the quantity [2]

$$
W_{i f}=\frac{\hbar}{4 \pi m^{*} c^{2}}\left(\frac{e^{2}}{\hbar c}\right) w_{i f}^{2} F_{i f},
$$

in which $w_{i f}=\left(E_{i}-E_{f}\right) / \hbar$ is the transition frequency. 


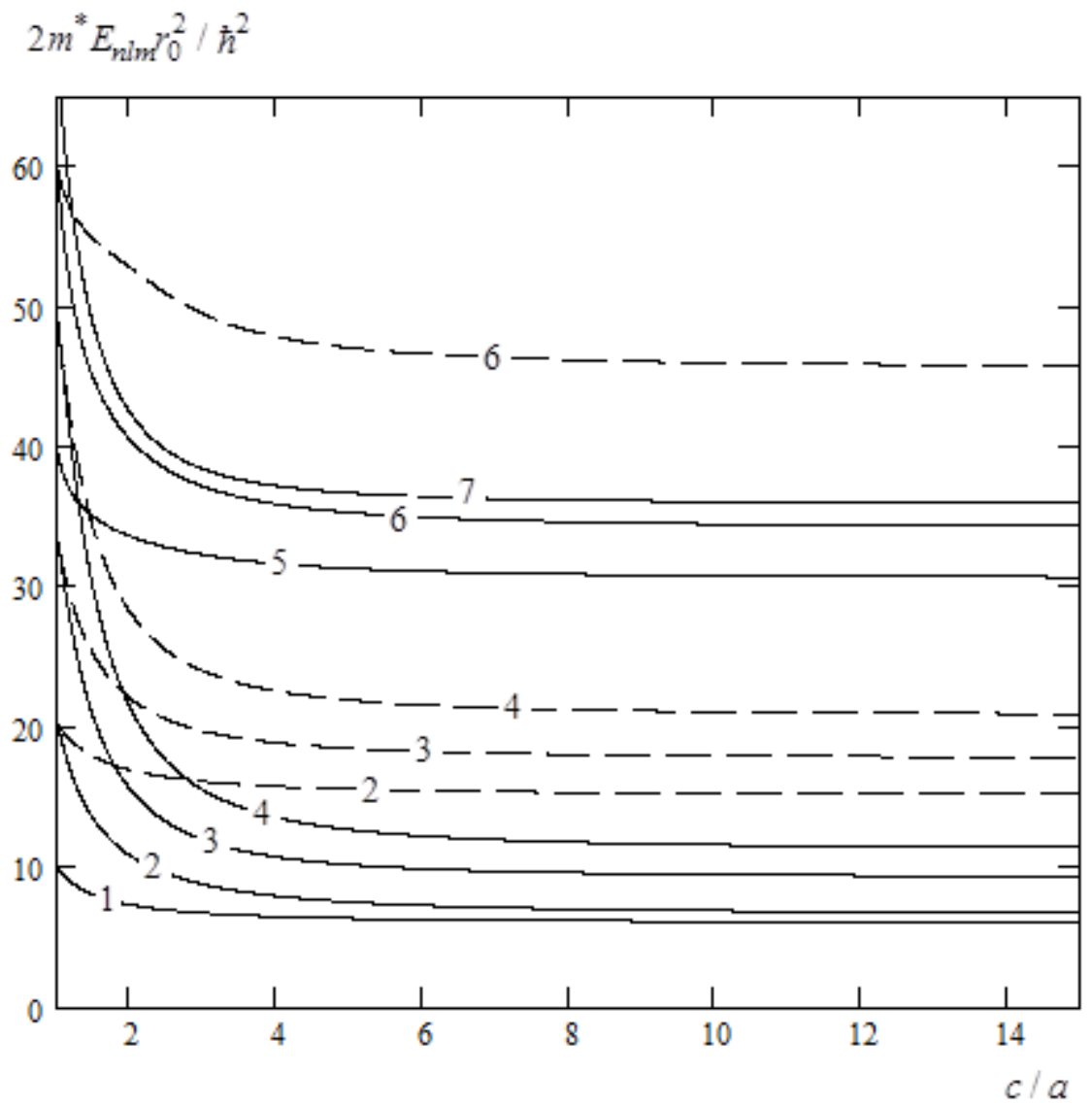

Fig. 1. Prolate ellipsoidal nanoparticle energy spectrum as a function of $c / a$. The energies are obtained by diagonalization of the Hamiltonian. The solid curves: $1-E_{100}, 2-E_{110}, 3-E_{120}, 4-E_{130}, 5-E_{200}, 6-E_{210}$, $7-E_{140}$. The dashed curves: $2-E_{111}, 3-E_{121}, 4-E_{131}, 6-E_{211}$. The energies are shown in units $\hbar^{2} /\left(2 m^{*} r_{0}^{2}\right)$.

\section{Results of calculation and discussion}

Equations (7) and (10), obtained above, enable us to express the dipole moment matrix elements as a function of shape-anisotropy parameter $c / a$. In Fig. 2 are shown the matrix elements obtained using Eq. (7) in which $a=r_{0}$ is fixed and $c$ increases from $c=a$ up to $c=2 a$. Fig. 2 shows that, when a deviation from the shape of a sphere is small, the matrix elements $\left\langle\Psi_{100}\left|d_{ \pm, z}\right| \Psi_{n l m}\right\rangle$ involving the ground state increase or decrease slightly when $c / a$ increases. The matrix elements corresponding to left and right polarization differ in sign. Matrix elements $\left\langle\Psi_{100}\left|d_{ \pm, z}\right| \Psi_{15 m}\right\rangle$ differ from zero in the second order of perturbation theory; they are thus not shown in Fig. 2.

The matrix elements calculated making use of Eq. (10) with $a=r_{0}$ are shown in Fig. 3 as a function of parameter $c / a$. To facilitate comparison, the states are specified with the same quantum numbers as in Fig. 2. Figure 3 clearly demonstrates that, when a deviation from the sphere is not small, the matrix elements are not monotonic functions of $c / a$. An interesting fact is the existence of both nodes and minima and maxima in the dependence of some matrix elements on $c / a$. Moreover, the numerical calculations revealed that matrix elements $\left\langle\Psi_{100}\left|d_{ \pm}\right| \Psi_{21 \mp 1}\right\rangle$ have discontinuity for $c / a=3.28$ (are not shown in Fig. 3). Here we do not investigate a cause of this discontinuity because the problem needs a separate consideration. 
Comparison shows that the matrix elements calculated using the Eqs. (7) and (10) coincide in the region of validity of the perturbation approximation, i.e. when $\mathrm{c} \sim \mathrm{a}$, whereas these matrix elements differ significantly when $c$ much exceeds $a$. In contrast to the energy levels for which the perturbation approximation is valid in the wide range of $c / a$ [19], the region of validity of Eq. (5) obtained for the wave functions using perturbation theory is thus substantially restricted.

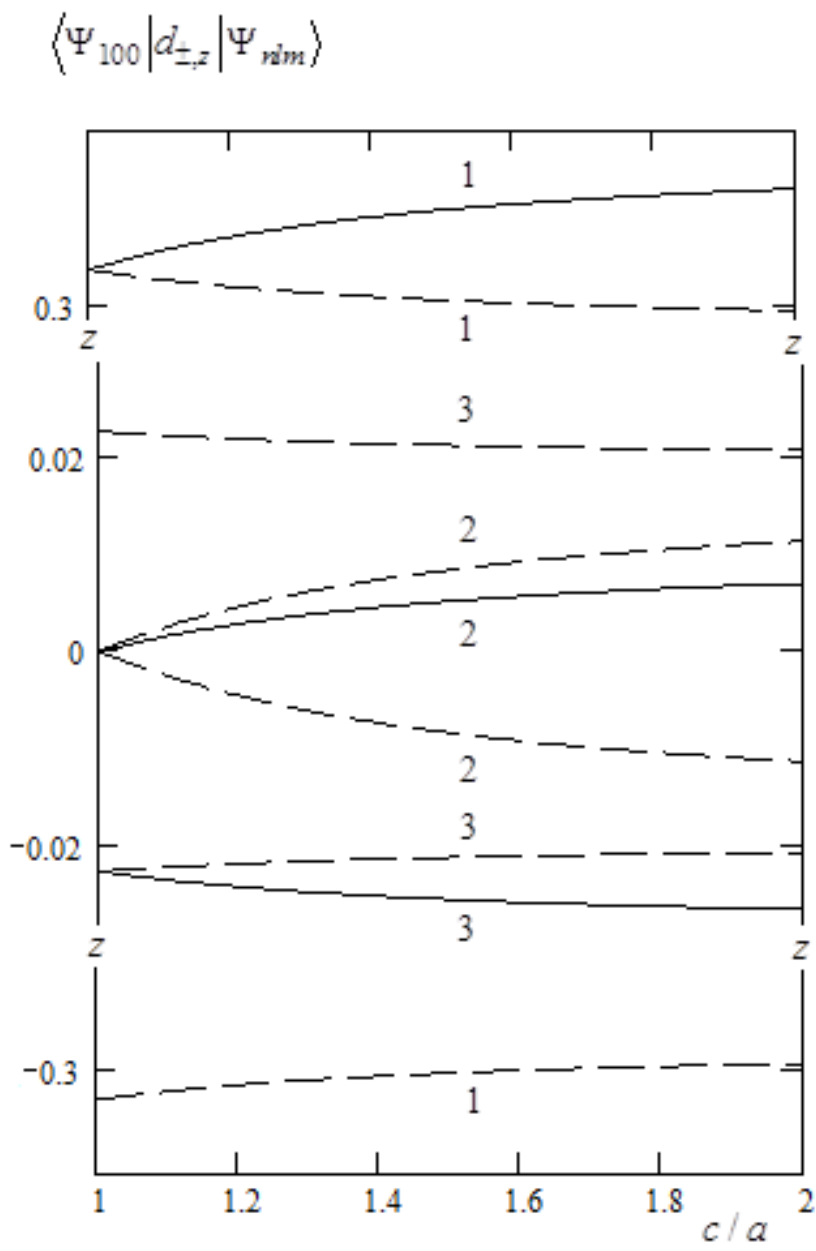

Fig. 2. The dipole moment matrix elements as a function of $c / a$ calculated using Eq. (7) with $a=r_{0}$. The solid curves:

$1-\left\langle\Psi_{100}\left|d_{z}\right| \Psi_{110}\right\rangle, 2-\left\langle\Psi_{100}\left|d_{z}\right| \Psi_{130}\right\rangle, 3-\left\langle\Psi_{100}\left|d_{z}\right| \Psi_{210}\right\rangle$.

The dashed curves: $1-\left\langle\Psi_{100}\left|d_{ \pm}\right| \Psi_{11 \mp 1}\right\rangle, 2-\left\langle\Psi_{100}\left|d_{ \pm}\right| \Psi_{13 \mp 1}\right\rangle$, $3-\left\langle\Psi_{100}\left|d_{ \pm}\right| \Psi_{21 \mp 1}\right\rangle$.

In [7] the dipole moment was expressed in terms of a momentum operator, and the transitionmatrix elements were calculated for an arbitrary deviation of the ellipsoid from a spherical shape. The interpolation formulae were obtained for the transition-matrix elements involving the ground and excited states $|110\rangle$ and $|11 \mp 1\rangle$. To compare our results with the results obtained in [7], we use the relation $|\langle f|\vec{p}| i\rangle|=m^{*} w_{i f}|\langle f|\vec{r}| i\rangle|$. We find good agreement for $\mathrm{c} \sim \mathrm{a}$ and satisfactory agreement for a large deviation from the spherical shape. The fact that our matrix element $\left\langle\Psi_{100}\left|d_{z}\right| \Psi_{110}\right\rangle$ is smaller, we attribute to the increasing contribution of the excited states in the transition-matrix element for $c / a>1.5$. The confirmation is that, if the sum rule for oscillator strengths is performed for the transitions from ground state $|100\rangle$ to four excited states $|110\rangle,|130\rangle,|210\rangle$ and $|150\rangle$, the value 
0.99 is obtained for $c \sim a$, whereas this value becomes about 0.60 for $c=1.5 a$. For transitions $|100\rangle \rightarrow|n l m\rangle$ with $m=-1$ or +1 , the sum rule gives 0.99 and 0.90 for $c \sim a$ and $c=1.5 a$, respectively.

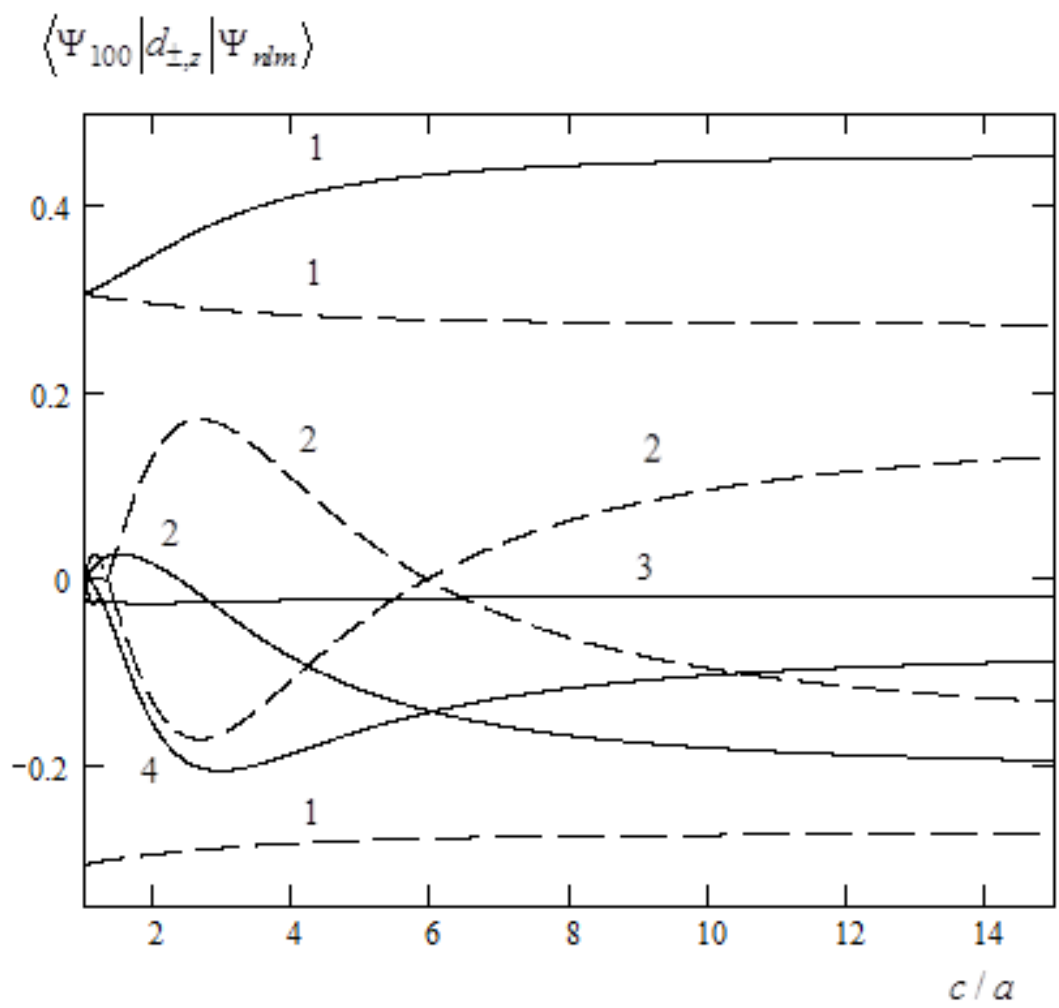

Fig. 3. The dipole moment matrix elements as a function of $c / a$ calculated using Eq. (10) with $a=r_{0}$. The solid curves: $1-$ $\left\langle\Psi_{100}\left|d_{z}\right| \Psi_{110}\right\rangle, 2-\left\langle\Psi_{100}\left|d_{z}\right| \Psi_{130}\right\rangle$ multiplied by factor 3, $3-\left\langle\Psi_{100}\left|d_{z}\right| \Psi_{210}\right\rangle, 4-\left\langle\Psi_{100}\left|d_{z}\right| \Psi_{150}\right\rangle$ multiplied by factor 50 .

The dashed curves: $1-\left\langle\Psi_{100}\left|d_{ \pm}\right| \Psi_{11 \mp 1}\right\rangle, 2-\left\langle\Psi_{100}\left|d_{ \pm}\right| \Psi_{13 \mp 1}\right\rangle$ multiplied by factor 20 .

In Fig. 4 are shown the interband transition probabilities as a function of $c / a$ calculated for an ellipsoidally shaped nanoparticle of $\mathrm{ZnO}$ using Eq. (12). The small semi-axis of the ellipsoid is fixed at $a=r_{0}=1 \mathrm{~nm}$; the effective mass is $m^{*}=0.24 m_{e}$ with electron mass $m_{e}$. Figure 4 shows that the transition probabilities involving the ground and first excited states are monotonically decreasing functions, whereas the transition probabilities involving the highly excited states are undulating functions of shape-anisotropy parameter $c / a$. When $a=r_{0}=N n m(N=2,3, \ldots)$, the dependence of transition probabilities $W_{i f}$ on $c / a$ does not alter but quantities decrease $N^{4}$ times.

\section{Conclusion}

In this work we have analysed the optical properties of ellipsoidally shaped nanoparticles. The optical transition-matrix elements have been calculated in the dipole approximation as a function of shapeanisotropy parameter $c / a$ using perturbation theory and with a direct diagonalization of the appropriate Hamiltonian. The transition-matrix elements involving the ground and first excited states 
are monotonic functions of $c / a$, whereas matrix elements involving the highly excited states have zeros and extrema that are reflected in the behaviour of the corresponding transition probabilities. Somewhat unexpected is that some matrix elements have discontinuity; this fact needs a further investigation.

The transitions forbidden for a spherical quantum dot but allowed in ellipsoidal nanoparticles exhibit promising properties. Specifically, for a nanoparticle of $\mathrm{ZnO}$, the transition probability between the ground state and second odd excited state has a sharp maximum at $c / a=1.27$, which

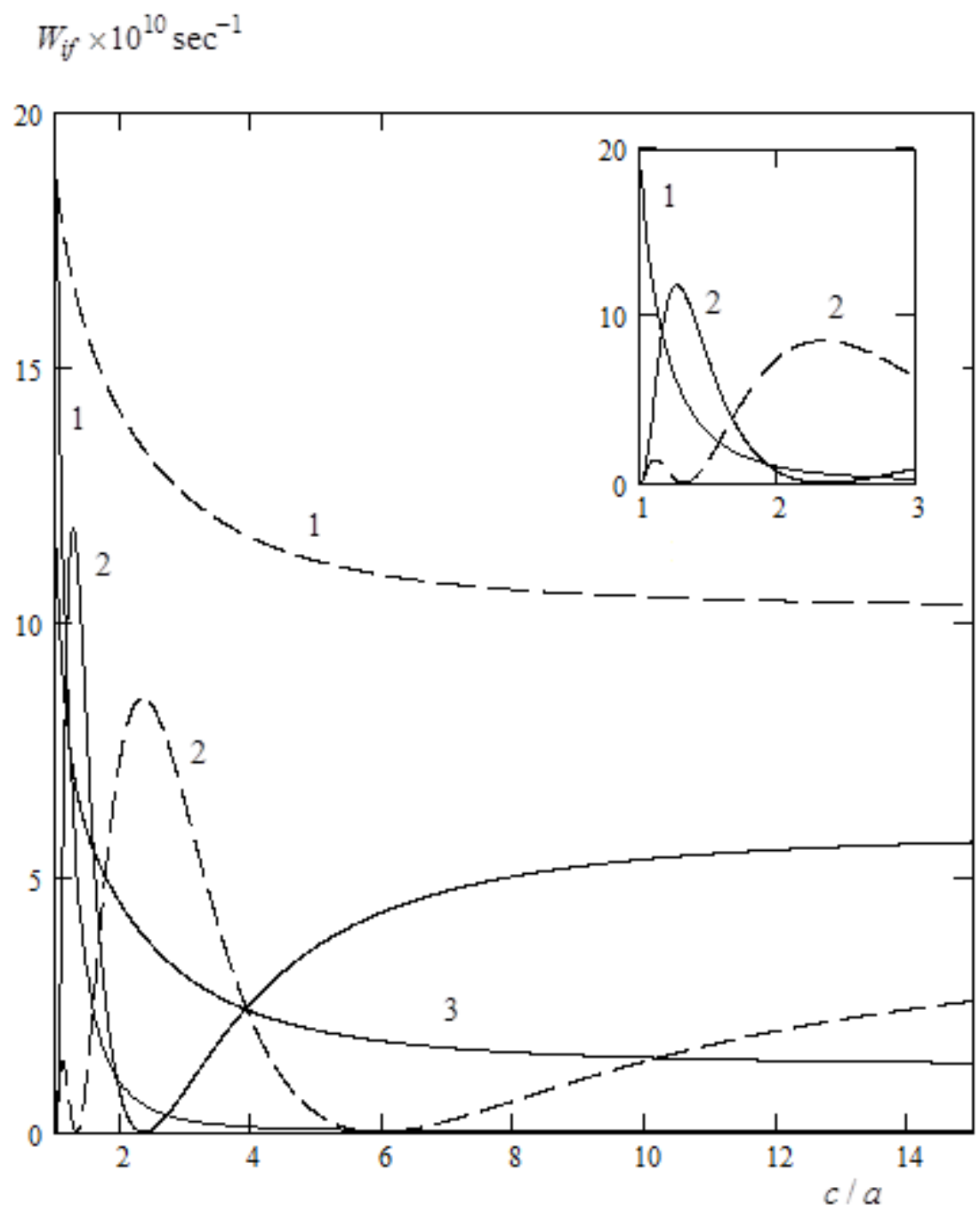

Fig. 4. The interband transition probabilities as a function of $c / a$ calculated for $\mathrm{ZnO}$ nanoparticle using Eq. (12) with $a=r_{0}=1 \mathrm{~nm}$. The solid curves: $1-W_{100,110}, 2-W_{100,130}$ multiplied by factor $50,3-W_{100,210}$. The dashed curves: $1-W_{100,11 \mp 1}, 2-W_{100,13 \mp 1}$ multiplied by factor 100 .

corresponds to absorption or emission of ultraviolet light for nanoparticle with $a=1 \mathrm{~nm}$. When the shape-anisotropy parameter increases, the transition probability becomes zero and then assumes a constant value when c>>a. Accordingly, the wavelength of absorbed or emitted light passes from the ultraviolet range of the electromagnetic spectrum to the infrared range. The transition probability between the ground state and first odd excited state has a maximum at $c=a$, which corresponds to an absorption or emission of infrared light, and monotonically decreases when $c / a$ increases. 
The present calculations thus confirm that emission and absorption processes can be tuned as a function of both the size and shape of a nanoparticle. In particular, nanoparticles with ellipsoidal shape can be grown with the infrared as well as ultraviolet features. Moreover, the variation of the shape-anisotropy parameter allows the realization of nanoparticles with emission and absorption spectra dependent on the polarization of the radiation. To observe the effect of undulation, one must measure the emission (or absorption) of radiation during the growth of a nanoparticle, which requires certain efforts due to a small magnitude of a transition probability.

\section{Acknowledgments}

This work was supported by Shota Rustavely Georgian National Science Foundation under the Grant DI/27/6-265/13.

\section{Appendix}

For the wave functions and energy levels of a charged particle confined in the ellipsoidal well, one can write in the second order of perturbation theory [19]

$$
\begin{aligned}
& \Psi_{n l m}=\psi_{n l m}+\alpha\left[A_{l+2} \sum_{n^{\prime}} \frac{\tau_{n l} \tau_{n^{\prime} l+2}}{\left(\tau_{n l}^{2}-\tau_{n^{\prime} l+2}^{2}\right)^{2}} \psi_{n^{\prime} l+2 m}\right. \\
& \left.+A_{l-2} \sum_{n^{\prime}} \frac{\tau_{n l} \tau_{n^{\prime} l-2}}{\left(\tau_{n l}^{2}-\tau_{n^{\prime} l-2}^{2}\right)^{2}} \psi_{n^{\prime} l-2 m}\right]+\alpha^{2} \Psi_{n l m}^{(2)}, \\
& E_{n l m}=\left[\frac{r_{0}^{2}}{a^{2}}+\alpha \frac{1-2 l(l+1)+2 m^{2}}{(2 l-1)(2 l+3)}\right] \varepsilon_{n l}+\alpha^{2} E_{n l m}^{(2)},
\end{aligned}
$$

where

$$
\begin{aligned}
& A_{l+2}=2(2 l+3) \sqrt{\frac{(l+1)^{2}-m^{2}}{4(l+1)^{2}-1}} \sqrt{\frac{(l+2)^{2}-m^{2}}{4(l+2)^{2}-1}}, \\
& A_{l-2}=-2(2 l-1) \sqrt{\frac{(l-1)^{2}-m^{2}}{4(l-1)^{2}-1}} \sqrt{\frac{l^{2}-m^{2}}{4 l^{2}-1}},
\end{aligned}
$$

and $\psi_{n l m}, \varepsilon_{n l}$ are the unperturbed wave functions and energy levels. We do not present here the explicit expressions for $\Psi_{n l m}^{(2)}$ and $E_{n l m}^{(2)}$ because they are cumbersome, but only note that $\Psi_{n l m}^{(2)}$ is a linear combination of unperturbed wave functions $\psi_{n l m}, \psi_{n l \pm 2 m}$ and $\psi_{n l \pm 4 m}$.

The expansion coefficients in (9) are defined with equations

$$
\begin{gathered}
C_{1 m}=\frac{\alpha U_{12}}{K_{i}^{2}-k_{1}^{2}-\alpha U_{11}} C_{2 m}, \\
C_{3 m}=\frac{\alpha U_{32}}{K_{i}^{2}-k_{3}^{2}-\alpha U_{33}} C_{2 m}, \\
C_{4 m}=\frac{\alpha U_{42}}{K_{i}^{2}-k_{4}^{2}-\alpha U_{44}} C_{2 m},
\end{gathered}
$$




$$
\begin{aligned}
& C_{2 m}=\left\{1+\alpha^{2}\left[\left(\frac{U_{12}}{K_{i}^{2}-k_{1}^{2}-\alpha U_{11}}\right)^{2}+\left(\frac{U_{32}}{K_{i}^{2}-k_{3}^{2}-\alpha U_{33}}\right)^{2}\right.\right. \\
& \left.\left.+\left(\frac{U_{42}}{K_{i}^{2}-k_{4}^{2}-\alpha U_{44}}\right)^{2}\right]\right\}^{-1 / 2},
\end{aligned}
$$

in which the superscripts $(g)$ and $(u)$ are omitted and $K_{i}^{2}=2 m^{*} E_{i} / \hbar^{2}$. In Eqs. (A.4) $k_{1}^{2}=\tau_{10}^{2} / r_{0}^{2}$, $k_{3}^{2}=\tau_{20}^{2} / r_{0}^{2}, k_{4}^{2}=\tau_{14}^{2} / r_{0}^{2}$ and $U_{12}=\left\langle\psi_{100}|U| \psi_{120}\right\rangle, \quad U_{32}=\left\langle\psi_{200}|U| \psi_{120}\right\rangle, U_{42}=\left\langle\psi_{12 m}|U| \psi_{12 m}\right\rangle$ for the even states and $k_{1}^{2}=\tau_{11}^{2} / r_{0}^{2}, \quad k_{3}^{2}=\tau_{21}^{2} / r_{0}^{2}, \quad k_{4}^{2}=\tau_{15}^{2} / r_{0}^{2} \quad$ and $U_{12}=\left\langle\psi_{11 m}|U| \psi_{13 m}\right\rangle$, $U_{32}=\left\langle\psi_{21 m}|U| \psi_{13 m}\right\rangle, U_{42}=\left\langle\psi_{15 m}|U| \psi_{13 m}\right\rangle$ for the odd states. The explicit expressions for $E_{i}$ are presented in Appendix B of Ref. [19].

\section{References}

[1] P. Harrison, Quantum Well, Wires and Dots, Wiley, New York, 2005.

[2] T. Chakraborty, Quantum Dots: A Survey of the Properties of Artificial Atoms, Elsevier Science, Amsterdam,1999.

[3] Al. L. Efros, A. L. Efros, Sov. Phys. Semiconductors, 16 (1982) 772.

[4] Al. L. Efros, A. V. Rodina, Phys.Rev. B 47 (1993) 10005.

[5] G. Cantele, D. Ninno, G. Iadonisi, Nano Letter 1 (2001) 121.

[6] G. Cantele, D. Ninno, G. Iadonisi, Phys. Rev. B 64 (2001) 125325.

[7] G. Cantele, G. Piacente, D. Ninno, G. Iadonisi, Phys. Rev. B 66 (2002) 113308.

[8] A. Shabaev, Al. L. Efros, Nano Letter, 4 (2004) 1821.

[9] H. Leon, J. L. Marin, R. Riera, Physca E 27 (2005) 385.

[10] A. Bagga, S. Ghosh, P. K. Chattopadhyay, Nanotechnology 16 (2005) 2726.

[11] W. S. Ferreira, J. S. de Sousa, J. A. K. Freire, G. A. Farias, V. N. Freire, Brazilian Journal of Physics 36 (2006) 438.

[12] K. G. Dvoyan, D. B. Hayapetyan, E. M. Kazaryan, A. A. Tshantshapanyan, Nanoscale Res. Lett. 2 (2007) 601.

[13] K. G. Dvoyan, D. B. Hayrapetian, E. M. Kazaryan, Nanoscale Res. Lett. 4 (2009) 106.

[14] K. Warda, J. Phys.: Condens. Matter 21 (2009) 345301.

[15] A. A. Gusev, O. Chuluunbaatar, S. I. Vinitsky, K. G. Dvoyan, E. M. Kazaryan, H. A. Sarkisyan, V. L. Debrov, A. S. Klobotskaya, V. V. Serov, Yad. Fiz. 75 (2012) 1281.

[16] M. Kuno, Introductory Nanoscience, Garland Science, London and New York, 2012.

[17] V. P. Dziuba, Yu. N. Kulchin, V. A. Milichko, Fiz. Tverd. Tela 56 (2014) 355.

[18] D. A. Baghdasaryan, D. B. Hayrapetyan, E. M. Kazaryan, Physica B (2015) 47985.

[19] T. Kereselidze, T. Tchelidze, R. Ya. Keserashvili, Physica E 68 (2015) 65.

[20] T. Kereselidze, T. Tchelidze, T, Nadareishvili, R. Ya. Keserashvili, Physica E 81 (2016) 196.

[21] D. Tobia, E. De Biasi, M. Granada, H. E. Troiani, G. Zampieri, E. Winkler and R. D. Zysler, J. Appl. Phys. 106 (2009) 103920.

[22] L.D. Landau, E.M. Lifshitz, Quantum mechanics: Non-Relativistic Theory, Elsevier, Singapore, 2007.

[23] A. G. Kurosh, Course of Higher Algebra, Moscow, Gostekhizdat, 1952 (in Russian).

[24] H. B. Dwight, Tables of Integrals and Other Mathematical Data, New York, the MacMillan Company, 1961.

[25] N. A. Bethe and E. E. Salpeter, Quantum Mechanics of One and Two-Electron Atoms, SpringerVerlag, 1957. 